\title{
Study on Recognition of the Relative Core Competence for Enterprise
}

\author{
Ting Wang ${ }^{1}$ \\ ${ }^{1}$ North China Electric Power University, \\ Baoding 071000, China
}

\author{
Hao Yuan ${ }^{1}$ \\ ${ }^{1}$ North China Electric Power University, \\ Baoding 071000, China
}

\begin{abstract}
The identification and evaluation of enterprise core competence is a prerequisite for building and improving enterprise competitive advantage ability. How to identify the enterprises core competence ? $^{[1]}$ The traditional research method is mainly identify the enterprise competitive advantage through the qualitative method. Now, along with the research, many scholars use a lot of quantitative methods to evaluate and identify the competitive advantages of the enterprise such as fuzzy comprehensive evaluation, analytic hierarchy process, value engineering and so on.However, many quantitative methods are rough and can't guarantee the accuracy of the results. The main direction of this paper is identify the value of the enterprise and improve the potentially valuable part through the most adoptable area method and value engineering(VE).
\end{abstract}

Keywords:the most adoptable area method; enterprises core competence; VE

\section{Introduction}

Core Competence is a special capability, resource and features with not easy to imitate. These make a great effects on the operation of company. In the value activity of value chain,the resource is helpful in distinction of each company. Core competence is a relative and dynamic capacity but not absolute or static which change itself with the variation of environmental policies, competitors and its own conditions.

We must establish the evaluation index to evaluate the core competence; but different enterprise need different evaluation index because of the nature of enterprise; so this increases the difficulty of designing the index system. ${ }^{[6]}$ Porter's competitiveness model analyze the competitiveness of enterprises and threats in an industrial chain from a supplier to a competitor and last to the consumer. Porter Model consider the external environment and market too much; not fully take into account the factors of their own for many small and medium-sized enterprises; they don't have the advantage in technology, channel, marketing and market share compared with competitors; they can't become an industry leader or followers. However, companies can't give up these existing capacity in the actual situation; these are the source of corporate profits; enterprise need the relative important ability of their own for needs of the market. Value chain model analyze the competitive strengths and weaknesses from the enterprise's own operations. ${ }^{[7]}$ The study focused on the business process; this is not applicable to some enterprises such as large-scale industry. The condition difference such as technology, resources and market share between enterprises is small; so enterprises pay more attention to corporate management culture and brand building, for example: total quality management, learning organization, innovation culture and so on. The ultimate aim to the identification of enterprise core competence is to improve irrational factors and optimize economic benefits.

This article make a discussion the core competence of enterprise; and take measures to analyze the competitive advantage in value engineering.

\section{To Assess Enterprise Core Competence with Value Engineering}

In the enterprise value chain, it is inevitable that cost and value is different in each part. It is necessary for enterprise to make a analysis and decision about how to choose a valuable focus on building the core part in business operations.This paper introduces a method about value engineering in the process to identify enterprise core competence.Value engineering is one of the organization's activities focused on functional analysis that spent lower total cost actualizing the necessary function of products and services. ${ }^{[2]}$

The basic process of Value Engineering is as follows:

\subsection{Solving function coefficient}

First, the analysis of the object to be selected are listed in a matrix form on the table; Then according to the importance of each function in the functional areas; we do one to one comparison. The relative importance of 1 point, a relatively minor 0 points; compared itself and referred to as " 0 " or " 1 ", here we referred to as " $1 " .{ }^{[3]}$ 
We can obtain the feature score through plusing the rampant scores. Using f1 represent the fraction of each function, and C $\mathrm{f}_{1}$ score represents the sum of the function; the function coefficients for each function is $F_{1}=f_{1} / C f_{1}$. Sum function coefficient of 1.0, function coefficients of each function is its importance in the functional areas quantified.

\subsection{Solving cost coefficient}

Find out the current cost of each function and mark $c_{1}$; Sum of total costs and mark $\Sigma c 1$. Using $C_{1}$ represent the cost in the function; so $\mathrm{C}_{1}=\mathrm{c}_{1} / \Sigma \mathrm{c}_{1}$. The sum of the cost coefficient of 1.0, the cost coefficient is the cost of each function in the function of the proportion of the total cost.

\subsection{Solving value coefficient}

The value coefficient is equal to the ratio of function coefficient and cost coefficient.In the data processing of value engineering; the bigger value coefficient is, the more important it is; the possibility of the core competence of the enterprise becomes bigger. A simple example will illustrate the point; we assume that the judgment matrix is obtained by following Delphy Technology; evaluating factors are as follows:

Technology and Product Development(TPD), Marketing and Sales(MS), Financial Management(FM), Channel Management(CM), Brand and Culture Construction(BCC), Production Quality and Safety(PQS), The Current Cost(TCC).

The sheet of data processing:

Fig.1
\begin{tabular}{|c|c|c|c|c|c|c|c|c|}
\hline Function & $\begin{array}{c}\text { TP } \\
\text { D }\end{array}$ & $\begin{array}{c}\text { M } \\
\text { S }\end{array}$ & $\begin{array}{c}\text { M } \\
\text { M }\end{array}$ & $\begin{array}{c}\text { CC } \\
\text { C }\end{array}$ & $\begin{array}{c}\text { CR } \\
\text { M }\end{array}$ & $\begin{array}{c}\text { PQ } \\
\text { S }\end{array}$ & $\begin{array}{c}\text { go } \\
\text { al }\end{array}$ \\
\hline TPD & 1 & 0 & 1 & 0 & 0 & 1 & 1 & 4 \\
\hline MS & 1 & 1 & 1 & 1 & 0 & 1 & 1 & 6 \\
\hline FM & 0 & 0 & 1 & 0 & 0 & 0 & 0 & 1 \\
\hline CM & 1 & 0 & 1 & 1 & 0 & 0 & 1 & 4 \\
\hline BCC & 1 & 1 & 1 & 1 & 1 & 1 & 1 & 7 \\
\hline CRM & 1 & 0 & 1 & 1 & 0 & 1 & 0 & 4 \\
\hline PQS & 1 & 0 & 1 & 0 & 0 & 1 & 1 & 4 \\
\hline
\end{tabular}

Fig.2
\begin{tabular}{|c|c|c|c|c|}
\hline Function & FC & TCC & CC & VC \\
\hline TPD & 0.129 & 40 & 0.111 & 1.162 \\
\hline MS & 0.194 & 100 & 0.278 & 0.698 \\
\hline FM & 0.032 & 30 & 0.083 & 0.386 \\
\hline CM & 0.129 & 50 & 0.139 & 0.928 \\
\hline BCC & 0.226 & 70 & 0.194 & 1.165 \\
\hline CRM & 0.129 & 40 & 0.111 & 1.162 \\
\hline PQS & 0.129 & 30 & 0.083 & 1.554 \\
\hline
\end{tabular}

We can know the production quality and safety is the most reasonable value chain activities by calculating; it is also a relative competitive advantage of operational factors.

\subsection{The defects of forced decision}

About the value coefficient,it only note the the deviation extent of 1 itself and select objects by size. While ignoring the size of value coefficient and the actual cost coefficient which can determine the degree of importance. If choose the value coefficient only noting the the deviation extent of 1 itself and select objects by size; The focus will be these objects that have a large deviation from 1 but the actual functional coefficient and actual cost factor is small; or excluding focus of some larger objects such as a factor which having a small deviation from 1 but the actual functional coefficient and actual cost factor is large.

\section{The Most Adoptable Area Method instead of Forced Decision}

Draw a map of the first quadrant in Cartesian coordinates in line (called the standard of value line). Every points which represent the objects fall in the standard of value line; the value of coefficient is equal to 1 . Illustrated the importance of their functions with the actual allocation of costs is proportionate, which generally do not chosen target value engineering; the value coefficient is greater than 1 (V>1); namely, the functional importance coefficient is greater than the actual cost coefficient. ${ }^{[4]}$

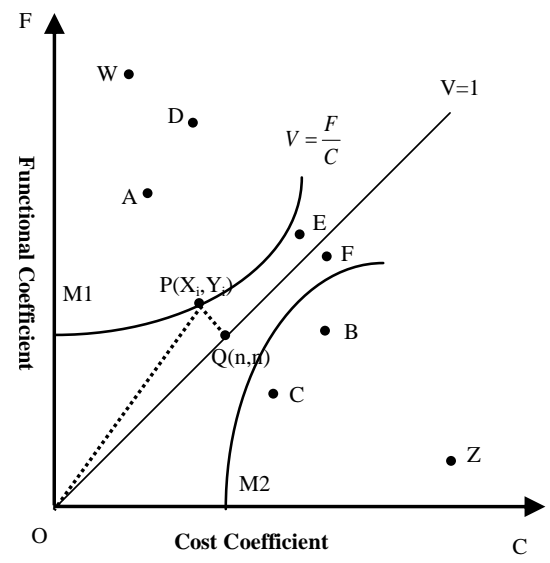

In the figure, the regional points fall in the area surrounded by two symmetrical curve such as E, F Could not be chosen as the object of value engineering. In contrast, the regional points fall out of two symmetrical curve such as A, B, C, D, W and $\mathrm{Z}$ should be chosen as the object of value engineering. The points between two symmetrical curve having a small deviation from 1 ; it is not worth increasing the cost to ensure the realization of its value.

Export curve equation of the most adoptable area method: located at any point on the curve $\mathrm{P}(\mathrm{xi}, \mathrm{yi})$, make a vertical line from point $\mathrm{P}$ to the standard value line; the foot point is $\mathrm{Q}$; because $\mathrm{Q}$ is in the the standard value line,the abscissa and ordinate are necessarily equal. Therefore tentatively make $\mathrm{Q}(\mathrm{n}, \mathrm{n})$ become the coordinates of foot point;connect $\mathrm{P}$ and $\mathrm{Q}$; the right triangle $\triangle \mathrm{PQO}$ is composed. ${ }^{[5]}$ 
Because

$$
\mathrm{PO}^{2}=\mathrm{PQ}^{2}+\mathrm{QO}^{2}, \mathrm{P}\left(\mathrm{X}_{\mathrm{i}}, \mathrm{Y}_{\mathrm{i}}\right), \mathrm{Q}(\mathrm{n}, \mathrm{n})
$$

So

$$
\begin{gathered}
\mathrm{x}_{i}^{2}+y_{i}^{2}=\left(x_{i}-n\right)^{2}+\left(y_{i}-n\right)^{2}+2 n^{2} \\
\Rightarrow \quad \mathrm{n}=0(\text { forsake }), \mathrm{n}=\frac{x_{i}+y_{i}}{2}
\end{gathered}
$$

If the distance of $\mathrm{PQ}$ and $\mathrm{QO}$ respectively is: $\mathrm{PQ}=\mathrm{R}, \mathrm{QO}=\mathrm{L}$; $\mathrm{B}$ is a constant; make $\mathrm{R} * \mathrm{~L}=\mathrm{B}$.

So M1 and M2 curve gradually move closer to $\mathrm{V}=1(\mathrm{M} 1$ and $\mathrm{M} 2$ are symmetrical about $\mathrm{V}=1$ ).

According to the formula, the distance $\mathrm{R}$ between point $\mathrm{P}$ and the straight line:

$$
R=\frac{\left|A x_{i}+B y_{i}+c\right|}{\sqrt{A^{2}+B^{2}}} \Rightarrow R=\frac{\left|x_{i}-y_{i}\right|}{\sqrt{2}}
$$

So

$$
\begin{aligned}
L & =\sqrt{\left(\frac{\mathrm{x}_{i}+y_{i}}{2}\right)^{2}+\left(\frac{\mathrm{x}_{i}+y_{i}}{2}\right)^{2}} \\
\Rightarrow L & =\frac{x_{i}+y_{i}}{\sqrt{2}} \text { or } L=-\frac{x_{i}+y_{i}}{\sqrt{2}} \text { (forsake) }
\end{aligned}
$$

$$
\text { And } \begin{aligned}
\mathrm{y}_{\mathrm{i}}^{2}-x_{i}^{2} & =2 B,\left(y_{i} \geq x_{i}\right) \\
& \Rightarrow \mathrm{y}_{i}=\sqrt{x_{i}+2 B}
\end{aligned}
$$

So

$$
\mathrm{x}_{i}=\sqrt{y_{i}+2 B},\left(\mathrm{x}_{i} \geq y_{i}\right)
$$

By determining the value of $B$, value engineering can be selected for improvement projects. This can help enterprise to adjust the value chain activities to improve the overall competitiveness and strengthening the relative competitive advantage.From the above graphics, we can see the curve gradually move closer to $\mathrm{V}=1$. In the physical truth, the distribution of B is certainly change with the environment, market, technology, society, resources and other conditions. We can assume that the value of B is substantially normal distribution. Enterprise must accumulate a lot of operational data in the long-term activities. Enterprise can choose a relatively good estimator by Delphi technique; the estimator must be around in the vicinity of the curve; In other words,closed to the line of $\mathrm{V}=1$. In the value activities, enterprise can find a point through self-assessment and relatively accurate; the point is closed to functional value and cost which can finely reflect the fluctuations of $\mathrm{B}$. The point $\mathrm{E}$, for instance. We can approximately deem the point on the curve; and get operational data in long-term activities. So we can acquire functional value and cost value of point $\mathrm{E}$; that is $\mathrm{B} \approx \mathrm{X}_{\mathrm{i}}(\mathrm{i}=1,2, \ldots, \mathrm{n})$. Thus we definite the position of the curve; and call interval estimation by pivot method. Then we need construct the unbiased and consistency hypothesis testing; on conditions of $\mathrm{T}$ distribution, we must guarantee hypothesis testing not can not reject the null. Simple as follows:

Assume

$$
\mathrm{B}_{1}=\mathrm{X}_{1}, \mathrm{~B}_{2}=\mathrm{X}_{2}, \ldots, \mathrm{B}_{\mathrm{n}}=\mathrm{X}_{\mathrm{n}}, \mathrm{X} \sim \mathrm{N}\left(\mu, \sigma^{2}\right)
$$

$$
\text { So } \quad \frac{\overline{\mathrm{x}}-\mu}{\mathrm{s} / \sqrt{\mathrm{n}}} \sim t(n-1)
$$

and

$$
\overline{\mathrm{x}}=\frac{1}{\mathrm{n}} \sum_{i=1}^{n} x_{i}, s=\frac{1}{n-1} \sum_{i=1}^{n}\left(x_{i}-\bar{x}\right)^{2}
$$

According to the moment estimation:

$$
E(X)=\frac{1}{\mathrm{n}} \sum_{i=1}^{n} x_{i}=\bar{x}
$$

So $\quad \hat{\mu}=\overline{\mathrm{x}}$

As the moment estimate of the parameters $\mu$ is $\bar{X}$, construct a function of $\overline{\mathrm{X}}_{\text {and } \mu} \mu$

$$
Z=\frac{\bar{x}-\mu}{s / \sqrt{n}} \sim t(n-1)
$$

So $\mathrm{Z}$ is the pivotal quantity.

For a given $\alpha(0<a<1)$, take a and $b(a<b)$, satisfying

$$
P\left\{a \leq \frac{\bar{x}-\mu}{s / \sqrt{n}} \leq b\right\}=1-\alpha, \forall \mu \in R
$$

Since the $\mathrm{T}$ distribution is a symmetrical distribution; to make the the shortest interval of average length, there should be

So

$$
\mathrm{P}\{\mathrm{Z}<\mathrm{a}\}=\mathrm{P}\{\mathrm{Z}>\mathrm{a}\}=\alpha / 2
$$

$$
a=-t_{\alpha / 2}(n-1), b=t_{\alpha / 2}(n-1)
$$

At the request of a confidence interval of $1-\alpha$ condition; we can't reject the null hypothesis

$$
\Rightarrow \frac{|\bar{x}-\mu|}{s / \sqrt{n}} \sim t(n-1) \Rightarrow-t_{\frac{\alpha}{2}} \leq \frac{|\bar{x}-\mu|}{s / \sqrt{n}} \leq t_{\frac{\alpha}{2}}
$$

Under the confidence interval of $1-\alpha$ condition; the confidence level of $\mu$ is

$$
\left[\overline{\mathrm{x}}-\frac{s}{\sqrt{n}} t_{\frac{\alpha}{2}}(n-1), \overline{\mathrm{x}}+\frac{s}{\sqrt{n}} t_{\frac{\alpha}{2}}(n-1)\right]
$$

So that it can determine the value range of $\mathrm{B}$.

By determining the point $\mathrm{B}$, we can get the complete curvilinear equation; the determination of curve position, we can basically certain what activity in the enterprise is efficient and most valuable. The point which is closer to the line $\mathrm{V}=1$ imply that the part is the core competitive 
advantage of the enterprise. Mean while, the point which is outside the curve have a strong potential for improvement. Through the improvement of these activities, it can enhance the enterprise's competition ability and guarantee the realization of the enterprise target.

\section{Conclusion}

Through the value engineering, we can mainly identify which are efficient value activities in the enterprise and which activities exist the possibility of improvement. But the value engineering can not guarantee the accuracy of comprehensive evaluation. Therefore, the article have changed a lot, further distinguished the evaluation object and refined evaluation objective area. So how to ensure the optimized range and guarantee the achievement of the subjective intent of decision-makers? This article can calculate the approximate range of optimization through mathematical statistics; the sample value of $E$ provided by enterprise can satisfied decision-makers' preferences and intentions. Through the above method, we perfectly improve the possibility that identify and reform the core competence.

\section{References}

[1] $\mathrm{Lu}$ Xinwen. The constitution, nurture and identification of core competence. Rural Economy, 2002, 2:26-28.

[2] Li Tingli. The project cost control of real estate development. Ms D Thesis. Beijing: Beijing Jiaotong University, 2007.

[3] Zhang Hongrui. Research on the cost management of water conservancy project. Ms D Thesis. Nanjing: Hohai University, 2008.

[4] Research on the cost management of central heating project. Ms D Thesis. Tianjing:Tianjin University, 2009.

[5] Ren Longyu. A theoretical problem of value engineering-Discussion of the most adoptable area method. Technical and Economic,1982, 5-10.

[6] Liu Guangsheng, Liu Shanshan, Cheng Zhihui. The accounting for core competence.Finance and Accounting, 2006(12):24-25.

[7] Yang Shufen. Study on the integration of enterprise value chain wiht core competence. Ms D Thesis.Wuhan: Wuhan University of Technology, 2009. 